\title{
ATOMIC LATTICES WITH UNIQUE COMPARABLE COMPLEMENTS
}

\author{
J. E. MCLAUGHLIN
}

1. Introduction. It follows immediately from the definitions that if a complemented lattice is modular then no element in the lattice can have distinct comparable complements. For lattices of finite dimension Dilworth [2] has demonstrated a converse...every complemented, nonmodular lattice of finite dimension contains a complemented nonmodular sublattice of order five. It is the purpose of this note to extend Dilworth's result to atomic lattices; the theorem proved may be stated as follows:

Every complemented, atomic lattice with unique comparable complements is modular.

An easy corollary of this is that every atomic lattice with unique complements is a Boolean algebra. This corollary improves the theorem of Birkhoff-Ward [1] stating that every complete atomic lattice with unique complements is a Boolean algebra.

It should be pointed out that the theorem of Dilworth [3] to the effect that every lattice is a sublattice of a lattice with unique complements shows that the word atomic cannot be deleted from the theorem stated above.

Finally, I would like to thank Professor Dilworth for raising the question settled here.

2. Notation and terminology. Lattice inclusion will be denoted by $a \supseteq b$, proper inclusion by $a \supset b$, covering by $a>b$. A lattice is said to be atomic if it has a null element, $z$, and if every non-null element contains an element covering $z$. An element $a^{\prime}$ is said to be a complement of $a$ if $a \cap a^{\prime}=z, a \cup a^{\prime}=u$, the unit element of the lattice. If every element of the lattice has a complement, the lattice is said to be complemented. For $a \supseteq b$ in a lattice the symbol $a / b$ denotes the sublattice of all $x$ with $a \supseteq x \supseteq b$.

3. Proof of the theorem. Throughout this section the lattice $L$ to which we refer is a complemented, atomic lattice in which $x \cup y$ $=x \cup w=u, x \cap y=x \bigcap w=z, y \supseteq w$ implies $y=w$, that is, comparable complements are unique. We tacitly assume $L$ has at least two elements. The proof that $L$ is modular is made by first showing that the

Received by the editors September 23, 1955. 
Dedekind transposition principle holds for one-dimensional quotients . $a>a \cap b$ if and only if $a \cup b>b$. This, together with the uniqueness of comparable complements, is then used to show that $L$ enjoys one of the essential properties of a projective geometry . . . Lemma 5. This in turn is used to show that comparable relative complements are unique and hence that $L$ is modular. I would like to thank the referee for pointing out a superfluous lemma in the original proof of the theorem.

LEMMA 1. If $p>z$ and $s=p \cup x \supset x$, then $u>x \cup t$ for any complement, $t$, of $s$.

It is sufficient to show that $x \cup_{t}$ is a complement of $p$, since comparable complements are unique. For this one observes that $p \cup(x \cup t)$ $=(p \cup x) \cup t=s \cup t=u$; therefore $p \cup(x \cup t) \supset x \cup t$, again since comparable complements are unique, and hence $p \cap(x \cup t)=z$.

COROLlaRY. For each $x \neq u$ in $L$ there exists an $m$ such that $u>m \supseteq x$.

LEмma 2. If $p>x \cap p=z$, then $p \cup x>x$.

Let $a=p \cup x$ and let $b$ be a complement of $a$. Then by Lemma 1, $u>x \cup b$. We first show that if $s \neq a$ is in $a / x$ then $t=a \cap(x \cup b) \supseteq s$. This shows in particular that $a>t$. Since $s \supseteq a, p \cup s=a$ and therefore $u>s \cup b$. But $s \cup b \supseteq x \cup b$ and $u>x \cup b$ implies $x \cup b=s \cup b$ implies $t \supseteq s$. Now choose a complement, $w$, of $t$. To prove the lemma it is sufficient to show that $w$ is also a complement of $x$. If $x \cup(a \cap w)=a$

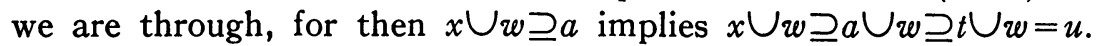
However, if $x \cup(a \cap w) \neq a$ then by our previous observation $t \supseteq x \cup(a \cap w)$ implies $t \supseteq a \cap w$ implies $a \cap w=z$. This supplies a contradiction in the form of $w$ having distinct comparable complements $a$ and $t$.

LEMMA 3. If $x \supset y$, then there exists $p>z$, such that $x \supseteq p, y \cap p=z$.

Suppose $y \supseteq p$ whenever $x \supseteq p>z$ and choose a complement, $v$, of $x \cap y$. Then $x \cap v \neq z$ and $p$ exists with $x \cap v \supseteq p>z$. Now $y \supseteq p$, a contradiction. This lemma says that any $x$ in $L$ is the union of all the points it contains . . . whether or not $L$ is complete.

Lemмa 4. The rule $a>a \cap b$ if and only if $a \cup b>b$ is valid in $L$.

If $a>a \cap b$, then by Lemma 3 there exists $p>z$ such that $a=p \cup(a \cap b)$. Then $a \cup b=p \cup(a \cap b) \cup b=p \cup b>b$ by Lemma 2. The corollary to Lemma 1 gives the dual of Lemma 3 , and this combined with the dual of Lemma 2 gives the opposite implication. 
LEMMA 5. If $a \neq u$ and $b$ is a complement of $a$ then for every $p>z$ in $L$

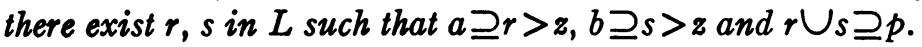

We may assume $a \cap p=b \cap p=z$ or there is nothing to prove. Then $p \cup a>a$ and by the duals of Lemmas 3 and $1, s=b \cap(p \cup a)>z$. Hence $(p \cup s) \cup a=p \cup a>a$ implies $p \cup s>(p \cup s) \cap a=r$ by the previous lemma. Therefore $p \cup r=p \cup s>p$ implies $r>r \cap p=z$ (again using Lemma 5). This proves the lemma.

LEMMA 6. If $a \supseteq b$, there exists an element $c$ such that $b \cup_{c}=a$ and $b \cap c=z$.

Choose any complement, $d$, of $b$ and put $c=a \bigcap d$. Combining Lemmas 3 and 5 it is easy to see that this $c$ has the desired properties.

TheOREM. $L$ is modular.

Proof. Suppose $e \supseteq f$ are relative complements of $b$ in $a / c$. Using Lemma 6 and its dual choose a relative complement, $x$, for $c$ in $b / z$ and a relative complement, $v$, for $a$ in $u / x$. Then $e$ and $f$ are both complements of $v$ and so $e=f$. This proves the theorem.

\section{BIBLIOGRAPHY}

1. G. Birkhoff and M. Ward, A characterization of Boolean algebras, Ann. of Math. vol. 40 (1939) pp. 609-610.

2. R. P. Dilworth, On complemented lattices, Tohoku Math. J. vol. 47 (1940) pp. 18-23.

3. - Lattices with unique complements, Trans. Amer. Math. Soc. vol. 57 (1945) pp. 123-154.

The University of Michigan 